Check for updates

Cite this: J. Mater. Chem. A, 2019, 7, 3083

Received 13th October 2018

Accepted 18th December 2018

DOI: $10.1039 / \mathrm{c} 8 \mathrm{ta0} 0981 \mathrm{c}$

rsc.li/materials-a

\section{Ethanolamine-assisted low-temperature crystallization of hydroxide nanoparticle ink into transparent and conductive ITO layers}

\begin{abstract}
Yujing Liu, (DD *a Thierry Moser, ${ }^{a}$ Christian Andres, ${ }^{a}$ Lovro Gorjan, ${ }^{b}$ Arndt Remhof, (D) ${ }^{c}$ Frank Clemens, ${ }^{b}$ Thomas Graule, ${ }^{b}$ Ayodhya N. Tiwari ${ }^{a}$ and Yaroslav E. Romanyuk (D) *a

Low-temperature crystallization is desired for achieving solution processable transparent conductive oxide (TCO) layers. Here we demonstrate a low-temperature crystallization of tin doped indium oxide (ITO) layers by taking preformed indium-tin hydroxide nanoparticles as the precursor and applying surface modification with ethanolamine on the as-prepared layers. Surface modification with ethanolamine is shown to speed up the dehydration of the hydroxide precursor, resulting in earlier onset of crystallization into the cubic bixbyite ITO phase with a larger crystal size. The ethanolamine-modified ITO nanoparticle layer possesses fewer surface hydroxyl groups as compared to the non-treated layer, corresponding to a higher charge carrier concentration, as manifested in a near IR absorption. The nanoparticle ITO layer shows dc conductivity two times higher than that of the non-treated layer. A conductivity of $43 \mathrm{~s} \mathrm{~cm}^{-1}$ combined with a high optical transmittance of above $90 \%$ in the $380-1100 \mathrm{~nm}$ wavelength range is obtained for the nanoparticle ITO layer annealed at $300{ }^{\circ} \mathrm{C}$.
\end{abstract}

\section{Introduction}

Transparent conducting oxides (TCOs) fabricated via vacuum sputtering techniques are widely applied as transparent electrodes in flat panel displays, touch panel displays, thin film solar cells and other opto-electronic devices. ${ }^{1-3}$ Wet-chemical processed TCOs, as a promising alternative to sputtered TCOs, have attracted a lot of attention due to their compatibility with roll-to-roll printing techniques and potential to be easily scaled up with less material waste. ${ }^{4-6}$ By far, the performance of TCO layers fabricated via wet-chemical approaches is still inferior to the ones fabricated by sputtering, with a sheet resistance of hundreds to thousands $\Omega$ per square. ${ }^{7-9}$

The use of preformed crystalline nanoparticles (NPs) is considered as one strategy towards conductive TCO layers. In the early studies by Ederth et al. individual ITO nanoparticles were found to have a resistivity as low as $2 \times 10^{-4} \Omega \mathrm{cm}$ on par with sputtered dense ITO films by analysing spectral optical reflectance and transmittance using effective medium theory. ${ }^{\mathbf{1 0 , 1 1}}$ However, the electrical conductivity of thin films

${ }^{a}$ Laboratory for Thin Films and Photovoltaics, Empa-Swiss Federal Laboratories for Materials Science \& Technology, Überlandstrasse 129, Dübendorf, CH-8600, Switzerland.E-mail: Yujing.Liu85@gmail.com; Yaroslav.Romanyuk@empa.ch ${ }^{b}$ Laboratory for High Performance Ceramics, Empa-Swiss Federal Laboratories for Materials Science \& Technology, Überlandstrasse 129, Dübendorf, CH-8600, Switzerland

${ }^{c}$ Laboratory for Materials for Energy Conversion, Empa-Swiss Federal Laboratories for Materials Science \& Technology, Überlandstrasse 129, Dübendorf, CH-8600, Switzerland processed from nanoparticle dispersion was much lower, on the order of $10^{-2} \Omega \mathrm{cm}$ after annealing at $800{ }^{\circ} \mathrm{C}$ due to separation between the micrometre-sized clusters of nanoparticles. Nanocrystals (NCs) of TCOs can be synthesized by different methods such as non-aqueous sol-gel ${ }^{12,13}$ or hot injection. ${ }^{7}$ In recent work by Song et al., ${ }^{7}$ ITO nanocrystals in a size of $11 \pm 2.4 \mathrm{~nm}$ were synthesized via a hot-injection synthesis and the processed thin film reached a sheet resistance of $112 \Omega \mathrm{sq}^{-1}$ with a transparency of $87 \%$ after 1 hour UV exposure and a post-treatment at $300{ }^{\circ} \mathrm{C}$ for 6 hours in a reducing atmosphere. One limitation of this approach is that a dispersing agent for steric stabilization is required to achieve a stable ink formulation. The use of the dispersing agent creates unwanted gaps between NCs, which impede the electron transport and decrease electrical conductivity. In recent work by Kim et al. ${ }^{\mathbf{1 4}}$ the gaps between adjacent nanoparticles were filled with precursors composed of nitrate, and the improvement of carrier mobility in the case of Ce-doped ITO layers was observed. However, the postdeposition annealing temperature was as high as $500{ }^{\circ} \mathrm{C}$.

Using molecular precursors such as metal salts or metal alkoxides is another wet-chemical approach to obtain TCO layers. It was reported that the resistivity of molecular precursor processed ITO films can be as low as $10^{-4} \Omega \mathrm{cm}$, approaching the values of sputtered ITO but a prolonged thermal annealing at $450-600{ }^{\circ} \mathrm{C}$ under a controlled atmosphere had to be applied. ${ }^{15-17}$ To facilitate the formation of metal oxide and the reduction of crystallization temperature, several strategies were proposed such as the tuning of precursor chemistry and use of chemical assisted annealing. ${ }^{18}$ Chemical combustion synthesis, 
a method utilizing the exothermic combustion of precursors to provide localized energy supply, was effective in reducing the processing temperature comparing with conventional methods. Following this method, the dc conductivity of ITO layers reached 130-140 S cm${ }^{-1}$ after annealing in hydrogen at a relatively low temperature of $250{ }^{\circ} \mathrm{C}$ or $300{ }^{\circ} \mathrm{C} .{ }^{19,20}$ However, the release of gas during the combustion of precursors limits this method mostly to very thin or/and amorphous layers.

Metal hydroxides are less explored precursors in the fabrication of conductive TCO layers, although the hydroxide precursor has been used in the synthesis of metal oxide nanocrystals, such as $\mathrm{TiO}_{2},{ }^{21} \mathrm{In}_{2} \mathrm{O}_{3}$ (ref. 22) and ITO. ${ }^{23}$ The phase transformation from metal hydroxide to oxide only involves a step of water condensation. In the case of undoped $\operatorname{In}_{2} \mathrm{O}_{3}$, the hydroxide phase can be transformed into the crystalline oxide at a mild temperature of $200-300{ }^{\circ} \mathrm{C} .{ }^{24,25}$

In this work, we explore the low temperature crystallization of conductive ITO layers based on inks composed of indium tin hydroxide (ITOH) nanoparticles. Surface modification with ethanolamine (ETA) molecules can reduce the phase transformation temperature to $\mathrm{ca} .250^{\circ} \mathrm{C}$ and promote crystallization into the bixbyite ITO phase. The assistance of ETA treatment in speeding up the dehydration of hydroxide, promoting the earlier crystallization as well as increase of crystal size after the same annealing is investigated with several complementary methods. An improved electrical conductivity of $43 \mathrm{~S} \mathrm{~cm}^{-1}$ combined with a high optical transmittance above $90 \%$ is demonstrated for nanoparticle-processed ITO layers annealed at $300{ }^{\circ} \mathrm{C}$.

\section{Experimental}

\subsection{Sample preparation}

Nanoparticle derived layers were fabricated via the spin coating of hydroxide nanoparticle inks. The sample preparation included four steps: (1) solvent thermal synthesis of nanoparticles and ink preparation, (2) spin-coating of nanoparticle ink in an $\mathrm{NH}_{3}$-containing atmosphere, (3) ETA modification of NP derived layers and (4) post-annealing of NP derived layers (with and without ETA modification) in forming gas $\left(95 \% \mathrm{~N}_{2} /\right.$ $5 \% \mathrm{H}_{2}$ ) at $300{ }^{\circ} \mathrm{C}$. A sequential order of the four steps is illustrated in Fig. 1.

Step (1): preparation of additive-free nanoparticle ink. The synthetic method of nanoparticles was modified from a previous publication. ${ }^{26}$ Indium tri-chloride $\left(\mathrm{InCl}_{3}\right.$, SigmaAldrich, 98\%) and tin tetra-chloride $\left(\mathrm{SnCl}_{4}\right.$, Sigma-Aldrich, nonaqueous, $98 \%$ ) were taken as metal precursors, while solid $\mathrm{NaOH}$ (granule, Sigma-Aldrich) as the catalyst and ethylene

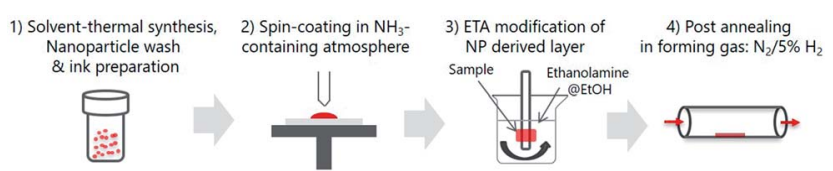

Fig. 1 Preparation procedure for nanoparticle derived layers with ETA modification. glycol (Carl Roth, 99\%) as the solvent. All the chemicals were used without further treatment. The molar ratio between $\mathrm{InCl}_{3}$ and $\mathrm{SnCl}_{4}$ was maintained as $9: 1$ (10\% doping), and a reaction temperature of $190{ }^{\circ} \mathrm{C}$ and a reaction time of 6 days were selected for the synthesis. The reacted nanoparticles were collected via centrifugation at $12000 \mathrm{rpm} \mathrm{min}{ }^{-1}$ for $15 \mathrm{~min}$ (Eppendorf, Centrifuge 5810R), and the collected nanoparticles were washed twice with distilled water to remove solvent residuals. To prepare nanoparticle ink, the collected nanoparticles (without drying) were re-dispersed in distilled water (DI) under magnetic stirring, without adding any stabilizing agents or organic additives. A series of inks with different solid loadings (concentrations) ranging from $2 \mathrm{wt} \%$ to $30 \mathrm{wt} \%$ could be prepared by adding the required amount of distilled water into the collected nanoparticles. The prepared inks are visually clear and free of sedimentation even after over 1 year storage at room temperature.

Step (2): spin coating under a controlled atmosphere. Spincoating was performed at room temperature, with a rotation speed of $2000 \mathrm{rpm} \mathrm{min}{ }^{-1}$ and a duration of 30 seconds for each layer. Soda-lime glasses, with a thickness of $1 \mathrm{~mm}$ and a size of $2.5 \times 2.5 \mathrm{~cm}$, were taken as substrates for depositing the prepared nanoparticle inks. They were cleaned in a consequential order with diluted acetic acid, isopropanol and DI water, and finished with oxygen plasma cleaning for $5 \mathrm{~min}$ to improve surface wettability. In order to achieve nanoparticle layers free of cracks and surface defects, ink concentration as well as the chamber atmosphere was optimized.

To minimize the presence of cracks in the obtained nanoparticle layer, ink concentrations varying from $30 \mathrm{wt} \%$ to $2 \mathrm{wt} \%$ were tested in a manner of single layer. With the optimized ink concentration ( $2 \mathrm{wt} \%)$, the spin-coating process was repeated multiple times to obtain the required thickness and a heating step at $80^{\circ} \mathrm{C}$ for $1 \mathrm{~min}$ was applied between every two adjacent layers. The $\mathrm{NH}_{3}$-containing chamber atmosphere was found effective in reducing surface defects such as streaks and comets and improving layer homogeneity. This atmosphere was generated by spin-coating $14 \mathrm{wt} \% \mathrm{NH}_{4} \mathrm{OH}$ water solution on a spare substrate, which was different from the substrates used for the spin-coating of nanoparticle layers. Afterwards, the spare substrate was taken out of the spin-coating chamber and the atmosphere was generated, followed by the fabrication of nanoparticle layers.

Step (3): ETA modification of nanoparticle derived layers. Ligand modification was performed on the as-deposited precursor layers before the final annealing step. Ethanolamine (ETA, Sigma-Aldrich, $\geq 98 \%$ ) was selected for ligand modification of hydroxide precursor layers. ETA was first dissolved in absolute ethanol with a concentration of $5 \mathrm{mM}$, and the samples were fixed in a Teflon sample holder and immersed in the solution for 24 hours. The solution was stirred at room temperature during the immersion time. The samples were rinsed with isopropanol continuously for $3 \mathrm{~min}$ after taking out from the ETA solution. We also attempted direct mixing of ligands into the nanoparticle ink; however, it caused 
agglomeration of nanoparticles and the spin-coated layers showed a much rougher surface.

Step (4): post annealing in forming gas. Nanoparticle layers with and without ETA modification were dried for $30 \mathrm{~min}$ on a preheated hot plate in air and then annealed for 3 hours in forming gas (mixture of $95 \% \mathrm{~N}_{2}$ and $5 \% \mathrm{H}_{2}$ ) at $300{ }^{\circ} \mathrm{C}$.

\subsection{Characterization techniques}

ITOH nanoparticle dispersions were characterized by dynamic light scattering (DLS) and zeta potential on a Zetasizer Nano series (Malvern). High resolution transmission electron microscopy (HRTEM) was performed using a field emission FET Titan 80-300 operated at $300 \mathrm{kV}$. Thermal gravimetric analysis (TGA) and differential scanning calorimetry (DSC) on nanoparticle powder were performed with a Netzsch STA 449 F3 Jupiter in synthetic air in a temperature range from room temperature to $600{ }^{\circ} \mathrm{C}$ at a ramping speed of $5{ }^{\circ} \mathrm{C}$ per min.

Thin layers before and after annealing were analysed by X-ray photoelectron spectroscopy (XPS) measurements on a Quantum 2000 from Physical Electronics with a monochromatic $\mathrm{Al} \mathrm{K} \alpha$ source $(1486.6 \mathrm{eV})$ operated at a base pressure below $8 \times 10^{-7}$ Pa. X-ray diffraction (XRD) analysis of nanoparticle layers was carried out to detect the phase transformation of nanoparticle layers with and without ETA modification along with the increase of annealing temperatures. The measurements were performed on a PANanalytical diffractometer (X'Pert Pro PW3040) equipped with Ni-filtered $\mathrm{Cu} \mathrm{K} \alpha$-radiation $(\lambda=1.5406$ A). In situ XRD measurements were conducted at temperatures of $200{ }^{\circ} \mathrm{C}, 225^{\circ} \mathrm{C}, 250{ }^{\circ} \mathrm{C}, 275^{\circ} \mathrm{C}$ and $300^{\circ} \mathrm{C}$, with a holding time of $30 \mathrm{~min}$ at each temperature. Transmittance was measured with a UV-Vis spectrometer Shimadzu UV-3600 from $200 \mathrm{~nm}$ to $2000 \mathrm{~nm}$ taking air as the reference (baseline). The electrical dc conductivity of annealed layers was measured by Hall-effect measurement on ECOPIA HMS3000 apparatus using the van der Pauw contacting configuration with gold contacts.

\section{Results and discussion}

\subsection{Fabrication and characterization of ITOH nanoparticles and ITOH layers}

The ITOH nanoparticles synthesized by this solvent-thermal approach at $190{ }^{\circ} \mathrm{C}$ exhibit a hexagonal structure, different from the cubic phase of bixbyite ITO. ${ }^{26}$ ITOH nanoparticles can be transferred into the crystalline cubic ITO phase at a mild temperature of $300{ }^{\circ} \mathrm{C}$ (in the form of dry powder), enabling their use as precursors or building blocks for various nanostructures of ITO electrodes. ${ }^{26}$ Another feature of such nanoparticles is their facile dispersibility in water, which facilitates the formation of water-based nanoparticle inks with long-term stability even at high solid loadings up to $30 \mathrm{wt} \%$ as tested in this work.

In Fig. 2, high resolution TEM images reveal the size of single nanoparticles as ca. $5 \mathrm{~nm}$, near spherical in shape, and crystalline in phase as visible from lattice fringes. Measurements with DLS show that the particles re-dispersed in DI-water are slightly agglomerated, with the peak of the distribution at 15-
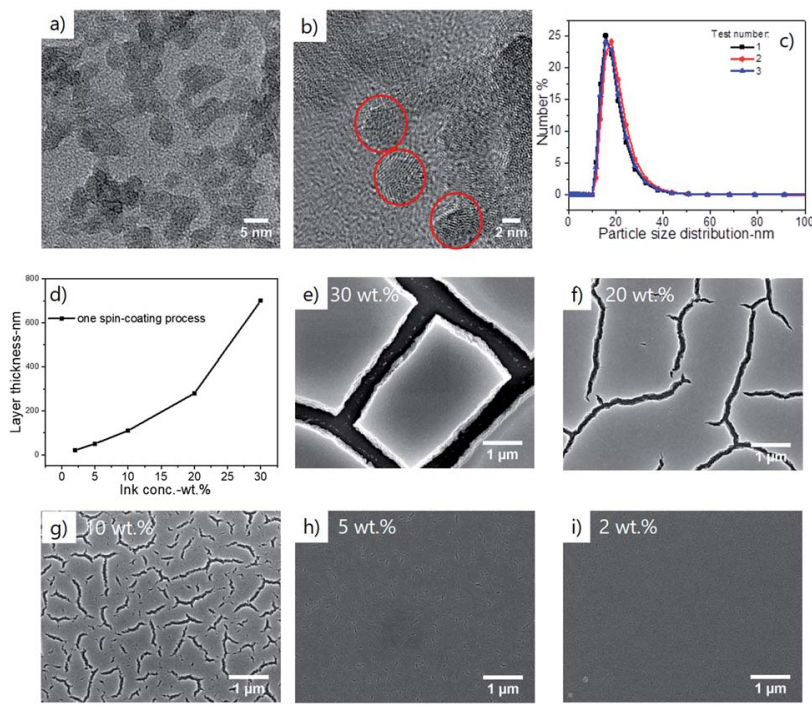

Fig. 2 ( $a$ and b) TEM morphology and (c) size distribution of solventthermal synthesized indium tin hydroxide nanoparticles re-dispersed in DI water (DLS); (d) the correlation between layer thickness and ink concentration; SEM top-views of spin-coated nanoparticle layers from (e) 30 wt\% ink, (f) 20 wt\%, (g) 10 wt\%, (h) 5 wt\% and (i) 2 wt\%.

$16 \mathrm{~nm}$ (Fig. 2c), indicating that the particles exist in clusters. The dispersion exhibits a zeta-potential of $+39 \mathrm{mV}$ and is very stable. The positive character of zeta potential is due to its adsorbed positively charged protons to counter near surface hydroxyl groups. No sedimentation is observed even after oneyear storage in a closed vial at room temperature. No additives or stabilizing agents were further added into the ink of indium tin hydroxide nanoparticles.

The thickness of one step spin-coated layer correlates with the ink concentration, as shown in Fig. 2d. Although a solid loading as high as $30 \mathrm{wt} \%$ can be achieved without stabilizing agents, the occurrence of cracks in fabricated nanoparticle layers was almost inevitable for inks with a high concentration (see Fig. 2e-i). To limit the crack formation, the ink concentration was decreased to $2 \mathrm{wt} \%$ and the thickness of a single spin-coating obtained layer was $c a .20 \mathrm{~nm}$. Therefore, a multiple spin-coating process was performed in order to achieve a nanoparticle layer with required thickness.

Besides the effect of ink concentration on the quality of coated layers, it was found out that the coating atmosphere also plays an important role, especially in avoiding the generation of surface defects such as 'streaks' and 'comets'. As reported by Redel $^{27}$ and Puzzo, ${ }^{28}$ streaks and comets are frequent defects in spin-coated nanoparticle layers because of particle agglomeration, dust particles in air, inappropriate setting of spin-coating parameters, etc. Our coating practice indicates that such defects can be avoided by generating a suitable atmosphere within the spin-coater chamber. In our case, an $\mathrm{NH}_{3}$-containing atmosphere has helped to reduce the number of defects such as comets/streaks and improve film homogeneity. The role of the $\mathrm{NH}_{3}$-containing atmosphere could be related to the surface characteristics of our hydroxide nanoparticles, which possess a positive zeta-potential of $+39 \mathrm{mV}$, indicating the existence of 
a positively charged surface double-layer. The spin-coating of a layer of $\mathrm{NH}_{4} \mathrm{OH}$ solution in the spin-coating chamber provided $\mathrm{NH}_{4}{ }^{+}$cations to the environment, and these cations could adsorb to the nanoparticle surface and further increase the surface charge of these nanoparticles. Consequentially, the nanoparticles were better stabilized and the number of surface defects due to nanoparticle agglomeration during the spincoating process was clearly reduced.

\subsection{The effect of ETA modification on the phase transformation of hydroxide into ITO}

We selected to conduct ETA modification on spin-coated precursor layers instead of mixing the solution of ETA with the ITOH nanoparticle ink. The reason behind this is that the addition of ETA into the nanoparticle ink inevitably caused the agglomeration of nanoparticles. The dispersion of ITOH nanoparticles shows a zeta-potential of $+39 \mathrm{mV}$ and the double layer of nanoparticles is positively charged due to adsorbed protons. Adding ETA (a Lewis base) into the nanoparticle ink can reverse the characteristics of the nanoparticle double layer and induce their agglomeration.

The effect of ETA modification is first revealed by the differences in oxygen 1s peaks of XPS measurement. As shown in Fig. 3a-d, the oxygen 1s peaks can be de-convoluted into two separate peaks: peak 1 is positioned at $\sim 530.0-530.5 \mathrm{eV}$ and corresponds to the metal-oxygen bonding, while peak 2 is positioned at $\sim 531.5-531.8 \mathrm{eV}$ and corresponds to the hydroxyl groups. The fitting results for non-treated and ETA-modified samples indicate that the area ratio of peak 2 increased from $42 \%$ to $55 \%$ for samples before the annealing, while it decreased from $25 \%$ to $15 \%$ for samples after the annealing. Apparently, the ETA modification has affected the phase transformation in two aspects: (1) it promoted a further
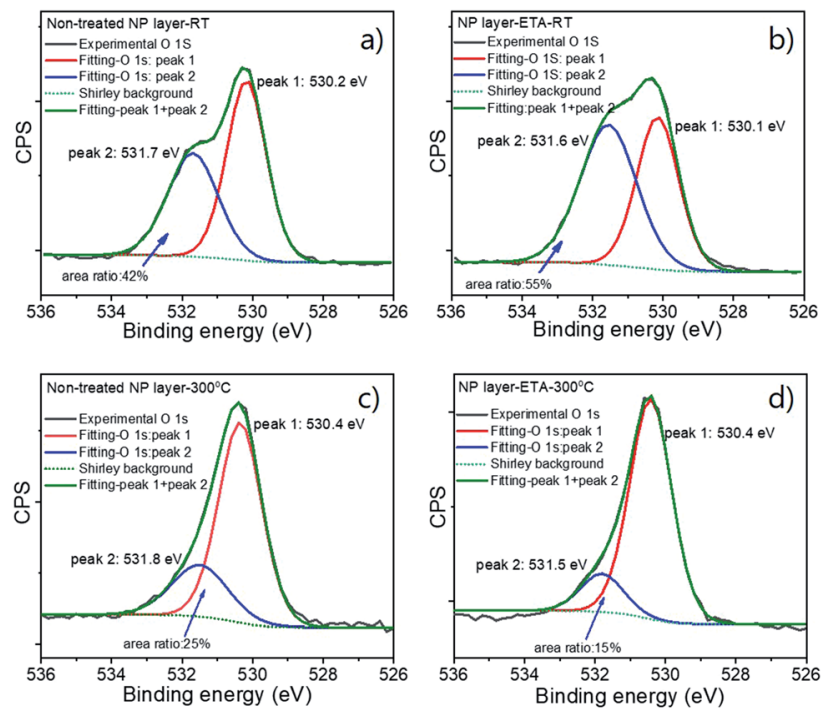

Fig. 3 XPS O 1s peak of nanoparticle derived layers (a) and (b) before annealing, (c) and (d) after annealing with and without ethanolamine modification. hydrolysis based on hydroxide nanoparticles before the annealing process (2) and resulted in a higher level of condensation or dehydration after the annealing process at $300{ }^{\circ} \mathrm{C}$, leading to a decreased number of hydroxyl groups.

It was also noticed that the loss of hydroxyl groups due to condensation or dehydration during the annealing process becomes quicker as compared to the non-treated sample. As shown in the DSC measurement in Fig. 4a and b, coupled with a higher exothermic peak, the weight loss at around $250{ }^{\circ} \mathrm{C}$ has a sharper slope comparing with the non-treated sample, indicating a faster removal of hydroxyl groups; consequently, completion of the phase transformation from hydroxide to oxide (step (3)) is shifted to a lower temperature. Besides, the amount of weight loss of steps (2) and (3) was also higher than that of the non-treated sample, with $18.1 \%$ against $17.0 \%(9.5 \%$ $+7.5 \%$ ), which implies that the sample with ETA modification possesses a larger number of hydroxyl groups before annealing, as already detected by XPS. After the completion of the phase transformation, the number of residual surface hydroxyl groups (indicated by step (4)) is lower for the ETA-treated sample, which is in agreement with XPS results. The sharp exothermal peak at $400{ }^{\circ} \mathrm{C}$ stems from the oxidation of the ETA residues which could be trapped by the nanoparticle powder after soaking in the ETA-ethanol solution (autoignition temperature of ETA is $410^{\circ} \mathrm{C}$ ). The ETA residues are not present in $300 \mathrm{~nm}$ thin ITOH layers because no traces of amino-groups were detected by XPS (not shown).

The shift of phase transformation to a lower temperature is also confirmed with in situ XRD measurement, shown as an earlier onset/appearance of the crystalline ITO phase under the same annealing conditions (Fig. 4c and d). Several studies on the phase transformation of indium hydroxide powder into the oxide phase reported the re-dissolution of indium hydroxide into an amorphous phase at $200-250{ }^{\circ} \mathrm{C}$ and the generation of a crystalline oxide phase from a re-nucleation process. ${ }^{23,26}$ In our case, no transformation of hydroxide into an amorphous phase
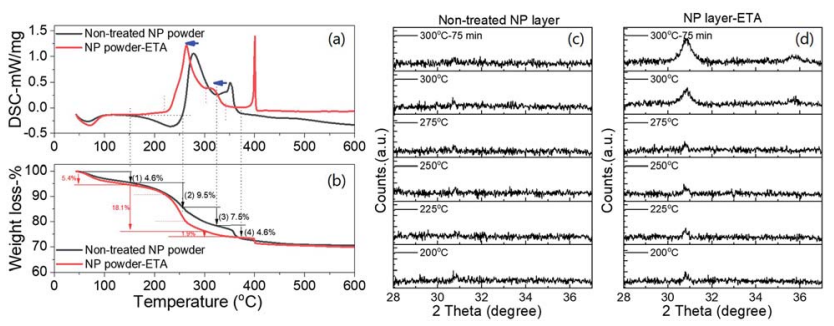

Fig. 4 Thermal analysis and in situ XRD measurement of nonannealed nanoparticle samples with and without ETA modification. The DSC/TG profiles in (a) and (b) show the transformation process, which can be divided into four steps: (1) evaporation of moisture before $150{ }^{\circ} \mathrm{C}$, shown as a weight loss of $4.6 \%$ and $5.4 \%$ for the nontreated sample and ETA modified sample; (2) the removal of hydrated water (adsorbed molecular water) and (3) phase transformation from hydroxide to oxide, which in total counts for $17.0 \%$ loss for the nontreated sample and $18.1 \%$ loss for the ETA modified sample; (4) removal of surface hydroxyl groups at higher temperatures, accounting for $4.6 \%$ for the non-treated sample and $1.9 \%$ for the ETA modified sample. The in situ XRD patterns of the (c) as-deposited nontreated NP layer and (d) ETA modified layer. 
was detected. We do not know whether this is because of the layer thickness restriction or from the ETA modification.

Amines have been reported to assist in the synthesis of metal oxides by complexing with metal ions and hindering their fast hydrolysis, i.e. acting as stabilizing ligands. ${ }^{23,29}$ In the present case, the ETA acts in a different way by speeding up the condensation/dehydration and inducing an earlier crystallization of the hydroxide ITOH precursor into the ITO phase. One hypothesis is that the surface of hydroxide nanoparticles gets functionalized with amino-containing ligands, which can be oxidized upon subsequent annealing in air. Through this exothermic reaction, energy is provided to hydroxide precursors (in a so-called combustion synthesis ${ }^{\mathbf{1 9 , 3 0}}$ ). Such a hypothesis of the combustion synthesis is supported by the stronger exothermal peak of the ETA-modified sample (Fig. 4a). However, no traces of amino-groups could be detected in the full spectrum XPS of non-annealed layers (not shown), so this effect cannot be dominant. A second mechanism could be related to the hydroxyl groups from ethanolamine molecules, which further promoted the hydrolysis of our as-prepared nanoparticle layers, seen as an increased number of hydroxyl groups compared with the reference sample in Fig. 3a and b, and finally resulted in a faster and higher level of dehydration/ condensation and earlier onset of crystallization. This effect has been reported in a recent work by Jao and others. ${ }^{31}$ With the presence of hydroxyl anions from tetramethylammonium hydroxide (TMAOH), the reaction rate for synthesizing NiO from nickel acetate was increased by two times, while the activation energy was decreased from $107 \mathrm{~kJ} \mathrm{~mol}^{-1}$ to $59 \mathrm{~kJ} \mathrm{~mol}^{-1}$.

\subsection{The effect of ETA modification on the conductive properties of nanoparticle ITO layers}

It can be expected that the ETA-modified nanoparticle layer exhibits a higher crystallinity and larger crystal size after annealing as compared to the non-treated layer. This is evidenced by XRD patterns in Fig. 5a. The grain size (or more precisely the crystal coherence length) calculated using the Debye-Scherrer formula from the full-width-at-half-maximum of (222), (400) and (440) reflexes is $35.3 \mathrm{~nm}, 34.3 \mathrm{~nm}$, and $31.9 \mathrm{~nm}$, which is larger than $30.5 \mathrm{~nm}, 29.4 \mathrm{~nm}$, and $28.7 \mathrm{~nm}$ for the non-treated sample. The top-view SEM images also reflect the difference of crystal sizes, as shown in Fig. 5b. The larger voids that appear in the top view SEM image of the ETA modified layers might be a consequence of crystal growth. ETA
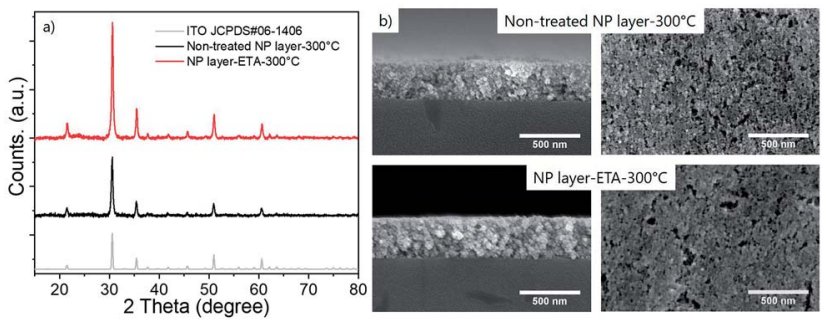

Fig. 5 (a) XRD and (b) SEM images of nanoparticle derived layers with and without ETA modification after the annealing process at $300^{\circ} \mathrm{C}$.
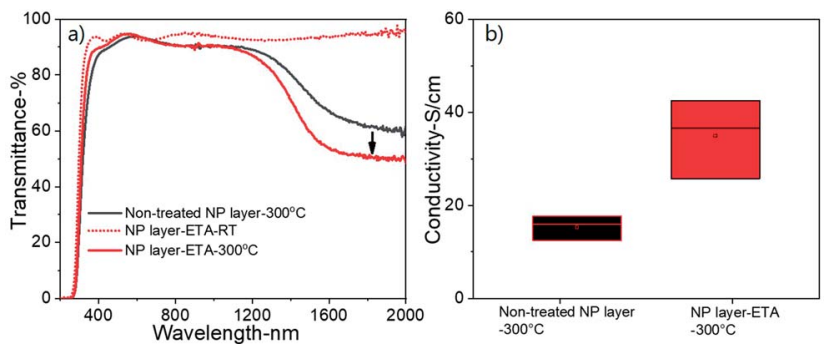

Fig. 6 (a) UV-Vis transmittance spectra and (b) dc conductivity of nanoparticle derived layers with and without ETA modification.

modification has no effect on the layer thickness, which is $310 \pm$ $10 \mathrm{~nm}$ for both samples.

The obtained ITO layers are transparent in the visible wavelength range, with a transmittance above $90 \%$ in the $380-$ $1100 \mathrm{~nm}$ wavelength range (Fig. 6a). In the IR range after $1100 \mathrm{~nm}$ the free carrier-induced IR absorption starts to appear and it is more pronounced for the ETA-modified layer. The difference in free carrier concentration can be explained by the different number of surface hydroxyl groups after the annealing process as measured by XPS. As proposed by Heiland and Morrison, ${ }^{32,33}$ the adsorbed oxygen species such as hydroxyl groups or hydrated water trap electrons from the surface region of n-type metal oxide particles and form a depletion layer. The existence of the depletion layer hinders the electron transport across the grain boundaries and also reduces carrier concentration. The experimental correlation between the presence of adsorbed surface states and the electrical conductive properties of nanoparticle-derived TCO layers has also been reported in recent studies from the Milliron group. ${ }^{8,34}$ In our case, a reduced number of surface hydroxyl groups correlate with a higher concentration of charge carriers, which also induces a shift of the UV cut-off to a shorter wavelength (the Burstein-Moss shift).

Electrical conductivity, a parameter correlated with layer crystallinity and charge carrier concentration, reflects the effect of ETA modification on the nanoparticle ITO layer. As plotted in Fig. $6 \mathrm{~b}$, the measured dc conductivities of ETA modified layers are two times higher than those of the non-treated layers. A high conductivity of $43 \mathrm{~S} \mathrm{~cm}^{-1}$ (sheet resistance of $782 \Omega \mathrm{sq}^{-1}$ ) has been measured for ETA modified layers annealed at $300{ }^{\circ} \mathrm{C}$ in forming gas of $5 \% \mathrm{H}_{2} / \mathrm{N}_{2}$. Compared with references, ${ }^{7,19}$ this is not the highest value for solution processed TCOs at a temperature of $300{ }^{\circ} \mathrm{C}$. However, an optimization of the modification process still holds potential for further reducing the crystallization temperature of the ITO phase and improvement of electrical properties.

\section{Conclusions}

Taking ITOH as a precursor, the fabrication of nanoparticle layers via multiple spin-coating of a stable additive-free hydroxide ink is demonstrated. Surface modification with ETA on a spin-coated nanoparticle layer can promote the phase transformation of hydroxide into the crystalline ITO phase, which results in an earlier onset of crystallization and larger 
crystal size after the same annealing. The ETA modification also results in fewer hydroxyl groups after the annealing process, thus yielding a higher charge carrier concentration and dc conductivity two times higher than that of the non-treated layer. A conductivity of $43 \mathrm{~S} \mathrm{~cm}^{-1}$ combined with an optical transmittance above $90 \%$ is demonstrated for an ITO layer processed from a dispersion of hydroxide nanoparticles.

\section{Conflicts of interest}

There are no conflicts to declare.

\section{Acknowledgements}

The authors acknowledge funding from the Swiss National Science Foundation (Grant IZLCZ2_170276/1) for a Sino-Swiss Science and Technology Cooperation project.

\section{References}

1 B. G. Lewis and D. C. Paine, Applications and Processing of Transparent Conducting Oxides, MRS Bull., 2011, 25(8), 2227.

$2 \mathrm{~K}$. Ellmer, Past achievements and future challenges in the development of optically transparent electrodes, Nat. Photonics, 2012, 6, 809.

3 D. S. Ginley and C. Bright, Transparent Conducting Oxides, MRS Bull., 2011, 25(8), 15-18.

4 R. M. Pasquarelli, D. S. Ginley and R. O'Hayre, Solution processing of transparent conductors: from flask to film, Chem. Soc. Rev., 2011, 40(11), 5406-5441.

5 J. Perelaer, et al., Printed electronics: the challenges involved in printing devices, interconnects, and contacts based on inorganic materials, J. Mater. Chem., 2010, 20(39), 84468453.

6 R. W. Schwartz, T. Schneller and R. Waser, Chemical solution deposition of electronic oxide films, C. R. Chim., 2004, 7(5), 433-461.

7 J. Song, et al., Back Cover: A General One-Pot Strategy for the Synthesis of High-Performance Transparent-ConductingOxide Nanocrystal Inks for All-Solution-Processed Devices, Angew. Chem., Int. Ed., 2015, 54(2), 698.

$8 \mathrm{~J}$. Ephraim, et al., Transparent Conductive Oxide Nanocrystals Coated with Insulators by Atomic Layer Deposition, Chem. Mater., 2016, 28(15), 5549-5553.

9 J.-Y. Kim and N. A. Kotov, Charge Transport Dilemma of Solution-Processed Nanomaterials, Chem. Mater., 2014, 26(1), 134-152.

$10 \mathrm{~J}$. Ederth, et al., Indium tin oxide films made from nanoparticles: models for the optical and electrical properties, Thin Solid Films, 2003, 445(2), 199-206.

$11 \mathrm{~J}$. Ederth, et al., Electrical and optical properties of thin films consisting of tin-doped indium oxide nanoparticles, Phys. Rev. B: Condens. Matter Mater. Phys., 2003, 68(15), 155410.

$12 \mathrm{~J}$. Ba, et al., Crystallization of indium tin oxide nanoparticles: from cooperative behavior to individuality, Small, 2007, 3(2), 310-317.
13 D. Ito, et al., Synthesis of Ligand-Stabilized Metal Oxide Nanocrystals and Epitaxial Core/Shell Nanocrystals via a Lower-Temperature Esterification Process, ACS Nano, 2014, 8(1), 64-75.

14 B. H. Kim, et al., High Mobility in Nanocrystal-Based Transparent Conducting Oxide Thin Films, ACS Nano, 2018, 12(4), 3200-3208.

$15 \mathrm{M}$. Toshiro and K. Akira, Indium-Tin Oxide Thin Films Prepared by Thermal Decomposition of Metallic Complex Salts, Jpn. J. Appl. Phys., Part 2, 1988, 27(10A), L1829.

$16 \mathrm{~S}$. Seki, et al., Highly conducting indium-tin-oxide transparent films prepared by dip-coating with an indium carboxylate salt, Surf. Coat. Technol., 2003, 169-170, 525-527.

17 R. B. H. Tahar, et al., Electronic transport in tin-doped indium oxide thin films prepared by sol-gel technique, $J$. Appl. Phys., 1998, 83(4), 2139-2141.

18 I. Bretos, et al., Low-temperature crystallization of solutionderived metal oxide thin films assisted by chemical processes, Chem. Soc. Rev., 2018, 47(2), 291-308.

19 M.-G. Kim, et al., Low-temperature fabrication of highperformance metal oxide thin-film electronics via combustion processing, Nat. Mater., 2011, 10, 382.

$20 \mathrm{X}$. Yu, et al., Spray-combustion synthesis: Efficient solution route to high-performance oxide transistors, Proc. Natl. Acad. Sci. U. S. A., 2015, 112(11), 3217-3222.

21 J.-N. Nian, et al., Structural Feature and Catalytic Performance of $\mathrm{Cu}$ Species Distributed over $\mathrm{TiO}_{2}$ Nanotubes, J. Phys. Chem. B, 2006, 110(51), 25817-25824.

22 S. Tang, et al., Effects of Heating Rate on the Nucleation, Growth, and Transformation of $\mathrm{InOOH}$ and $\mathrm{In}_{2} \mathrm{O}_{3}$ via Solvothermal Reactions, J. Phys. Chem. C, 2014, 118(36), 21170-21176.

$23 \mathrm{Z}$. Chen, et al., Ethanolamine-assisted synthesis of sizecontrolled indium tin oxide nanoinks for low temperature solution deposited transparent conductive films, J. Mater. Chem. C, 2015, 3(43), 11464-11470.

$24 \mathrm{X}$. Xu and X. Wang, Size- and Surface-Determined Transformations: From Ultrathin InOOH Nanowires to Uniform c- $\operatorname{In}_{2} \mathrm{O}_{3}$ Nanocubes and $r h-\mathrm{In}_{2} \mathrm{O}_{3}$ Nanowires, Inorg. Chem., 2009, 48(8), 3890-3895.

25 L. A. Pérez-Maqueda, L. Wang and E. Matijević, Nanosize Indium Hydroxide by Peptization of Colloidal Precipitates, Langmuir, 1998, 14(16), 4397-4401.

26 Y. Liu, et al., Assembly of mesoporous indium tin oxide electrodes from nano-hydroxide building blocks, Chem. Sci., 2012, 3(7), 2367-2374.

27 E. Redel, et al., Hierarchical Nanoparticle Bragg Mirrors: Tandem and Gradient Architectures, Small, 2011, 7(24), 3465-3471.

28 D. P. Puzzo, et al., Color from colorless nanomaterials: Bragg reflectors made of nanoparticles, J. Mater. Chem., 2009, 19(21), 3500-3506.

29 L. Znaidi, et al., Oriented ZnO thin films synthesis by sol-gel process for laser application, Thin Solid Films, 2003, 428(1), 257-262.

30 N. Martin-Arbella, et al., Photoactivation of Sol-Gel Precursors for the Low-Temperature Preparation of $\mathrm{PbTiO}_{3}$ 
Ferroelectric Thin Films, J. Am. Ceram. Soc., 2011, 94(2), 396403.

31 M. Jao, Low temperature and rapic formation of high quality metal oxide thin film via a hydroxide-assisted energy conservation strategy, J. Mater. Chem. C, 2018, 6, 9941-9949.

32 S. R. Morrison, Surface Barrier Effects in Adsorption, Illustrated by Zinc Oxide, in Advances in Catalysis, ed. W. G. Frankenburg, V. I. Komarewsky and E. K. Rideal, Academic Press, 1955, pp. 259-301.
33 G. Heiland, E. Mollwo, and F. Stöckmann, Electronic Processes in Zinc Oxide, in Solid State Physics, ed. F. Seitz and D. Turnbull, Academic Press, 1959, pp. 191-323.

34 C. M. Staller, et al., Tuning Nanocrystal Surface Depletion by Controlling Dopant Distribution as a Route Toward Enhanced Film Conductivity, Nano Lett., 2018, 18(5), 28702878. 